\title{
Automated Disaster Monitoring from Social Media Posts using Al based Location Intelligence and Sentiment Analysis
}

This paper was downloaded from TechRxiv (https://www.techrxiv.org).

LICENSE

CC BY 4.0

SUBMISSION DATE / POSTED DATE

22-02-2022 / 01-03-2022

\section{CITATION}

Sufi, Dr. Fahim; Khalil, Ibrahim (2022): Automated Disaster Monitoring from Social Media Posts using Al based Location Intelligence and Sentiment Analysis. TechRxiv. Preprint.

https://doi.org/10.36227/techrxiv.19212105.v1

DOI

10.36227/techrxiv.19212105.v1 


\title{
Automated Disaster Monitoring from Social Media Posts using AI based Location Intelligence and Sentiment Analysis
}

\author{
Fahim K. Sufi, Member, IEEE, and Ibrahim Khalil
}

\begin{abstract}
Worldwide disasters like bushfires, earthquakes, floods, cyclones, heatwaves etc. have affected the lives of social media users in an unprecedented manner. They are constantly posting their level of negativity over the disaster situations at their location of interest. Understanding location-oriented sentiments about disaster situation is of prime importance for political leaders, and strategic decision-makers. To this end, we present a new fully automated algorithm based on artificial intelligence (AI) and Natural Language Processing (NLP), for extraction of location-oriented public sentiments on global disaster situation. We designed the proposed system to obtain exhaustive knowledge and insights on social media feeds related to disaster in $\mathbf{1 1 0}$ languages through AI and NLP based sentiment analysis, named entity recognition (NER), anomaly detection, regression, and Getis Ord Gi* algorithms. We deployed and tested this algorithm on live Twitter feeds from 28 September 2021 till 6 October 2021. Tweets with 67,515 entities in 39 different languages were processed during this period. Our novel algorithm extracted 9727 location entities with greater than $\mathbf{7 0 \%}$ confidence from live twitter feed and displayed the locations of possible disasters with disaster intelligence. The rates of average precision, recall and F1-Score were measured to be $0.93,0.88$ and 0.90 respectively. Overall, the fully automated disaster monitoring solution demonstrated $97 \%$ accuracy. According to the best of our knowledge, this study is the first to report location intelligence with NER, sentiment analysis, regression and anomaly detection on social media messages related to disasters and has covered the largest set of languages.
\end{abstract}

Index Terms-Artificial Intelligence, Sentiment Analysis, Named Entity Recognition, Automated Location Extraction, AI Based Disaster Monitoring Dashboard, Disaster Intelligence Mobile App

\section{INTRODUCTION}

$\mathrm{N}$ ATURAL disasters are adverse events resulting from natural processes of the Earth like firestorms, dust storms, floods, hurricanes, tornadoes, volcanic eruptions, earthquakes, tsunamis, storms, and other geologic processes. Many of these events have aggressive negative effects on human life, infrastructure, economy, and society [1]. Average economic losses resulting from natural disasters worldwide has been over 308 billion USD from 2016 to 2020 [2]. This year, only Hurricane Ida caused 64.5-billion-dollar damage and 96 fatalities with power outages, landfalls, and

Fahim K Sufi, is with Federal Government, Melbourne, VIC-3000, Australia (e-mail: research@fahimsufi.com).

Ibrahim Khalil is with School of CS \& IT, RMIT University, Melbourne, VIC-3000, Australia (e-mail: Ibrahim.Khalil@rmit.edu.au). flash floods within just 4 days between $8^{\text {th }}$ Aug 2021 and $1^{\text {st }}$ September 2021 [3]. According to the most recent figures from World Bank, impact of major floods and earthquakes can reach 7 to 17 percent of a country's GDP, which could be avoided by investing in early warning system as well as strengthening disaster preparedness [4].

To reduce the adverse effect of natural disasters, it is vital to collect, aggregate, curate, analyze and comprehend disaster data. According to United Nations, the first element of efficient early warning systems is "Disaster risk knowledge based on the systematic collection of data and disaster risk assessments" [5]. Hence, in our most recent study, we have harnessed the power of AI to obtain hidden insight from NASA's global landslide incident data [6] [7]. While maintaining and managing a global disaster incident dataset is an extremely daunting task and only suitable for large enterprises and government entities (like NASA), harnessing social media data on disaster could provide a more sustainable approach. Hence, there have been a surge of research works on analyzing disaster related social media (e.g., Twitter) data for providing comprehensive situational awareness, conducting disaster risk assessment as well as boosting disaster preparedness [8] [9] [10] [11]. These studies harnessed several location extraction methods to estimate the possible location of the disasters [8] [9] [10] [11].

However, the existing works severely lack in providing accurate location intelligence since these studies used geotagged information of twitter [8] [10] [11]. Geo-tagged twitter were also used in [12] to better understand the emotions of theme park visitors, in [13] to comprehend user satisfaction on wireless services, and in [14] to understand political agendas. According to [9], tweets with geo-tagged location could be found only within $1 \%$ of the vast tweet messages. This means studies in [8] [10] [11] [12], [13], and [14] failed to utilize 99\% of tweet messages without location information (i.e., nongeotagged tweets). Other drawback of using geotagged tweets is that people tend to move around, and tweets can retain an old and invalid address of the user. Moreover, if location services are not enabled from the user end, research from tweet messages could result in flaw in analysis.

Because of the drawback of using geotagged tweet (i.e.,

Mentions of supplemental materials and animal/human rights statements can be included here.

Color versions of one or more of the figures in this article are available online at http://ieeexplore.ieee.org 
$>$ REPLACE THIS LINE WITH YOUR MANUSCRIPT ID NUMBER (DOUBLE-CLICK HERE TO EDIT) <

tweets with location information), researchers in [9], extracted possible location information from the tweet text by conducting a lookup search against a locally maintained location table. Even though, using lookup against the tweet text with location table, means utilizing the almost $100 \%$ of the tweet messages (as opposed to only $1 \%$ of geotagged tweets), it is only suitable for selected region of interest. If the method demonstrated in [9], is to be implemented globally across all the regions, then the requirement of managing a vast location table with all possible locations in the world becomes unsustainable for a small or medium sized organization.

Other than inefficient and ineffective location extraction mechanisms, existing research in [8] [9] [10] [11] [12] [13] [14] also suffers from the following:

- Limited Language Coverage: Existing studies provided situational awareness primarily from English tweets (with the exception of [11] that worked on Hindi tweets and [14] that worked on regional Indian languages).

- Limited Monitoring of Disasters: Existing studies reported only selected disasters (earthquake in [8], flood in [9], earthquake and plane crash in [10] etc.).

- Disregarding the Sentiment of Users: Not considering the users' sentiment results in classification errors, since adverse disasters and global events are associated with negative sentiments as shown in our latest studies [15] [16].

- Limited accessibility: Existing research are not suitable for a strategic decision maker who is willing to receive just-in-time and real-time disaster insights in wide range of platforms including iOS and Android mobile apps.

With these limitations in mind, this study is designed to use application programming interfaces (API) for all the major sense the all the major events for major disaster events like Bushfire, Cyclone, Drought, Earthquake, Flood, Hurricane, Landfall, Landslide, Tsunami and Volcano. As seen from Fig. 1 , after sensing any possible disaster from social media via API, the social media messages go through a preprocessing stage with AI and NLP based techniques like Named Entity Recognition (NER) [15] [17], sentiment analysis and category classification. This novel preprocessing stage reveals a new set of features (where sentiment information is used as one of the features) that are fed into deep learning system like Convolution Neural Network (CNN) based anomaly detection, automated regression and Getis-Ord Gi*. Since this novel methodology is the first of its kind that made use of social media sentiments as an input to deep learning with $\mathrm{CNN}$, the disaster detection accuracy is significantly higher than all existing studies reported in the literature [8] [9] [10] [11] [12] [13] [14]. Our comprehensive evaluation with 67,528 Tweets with 67,515 entities in 39 different languages on 10 different disaster types shows that precision achieved on disaster location classification with the proposed method is 0.93 as opposed to 0.60 to 0.80 precisions shown in [8] or 0.81 precision in [9]. Apart from higher level of disaster location classification accuracy, this study also harnesses the following benefits:

1. Works on a wide range of languages (more than 110 as per [15])

2. Works simultaneously in all regions across the globe and on a substantially wider range of disaster types (i.e., not only limited to the region of interest or limited number of disaster like [8] [9] [10])

3. Solution instantly accessible to the decision maker in a wide range of platforms including mobile (via both iOS and Android Apps), tablet and desktop

4. The hidden insights learned from deep learning methods like CNN is presented to the user in an easy-to-understand language utilizing state of the art NLP [18]

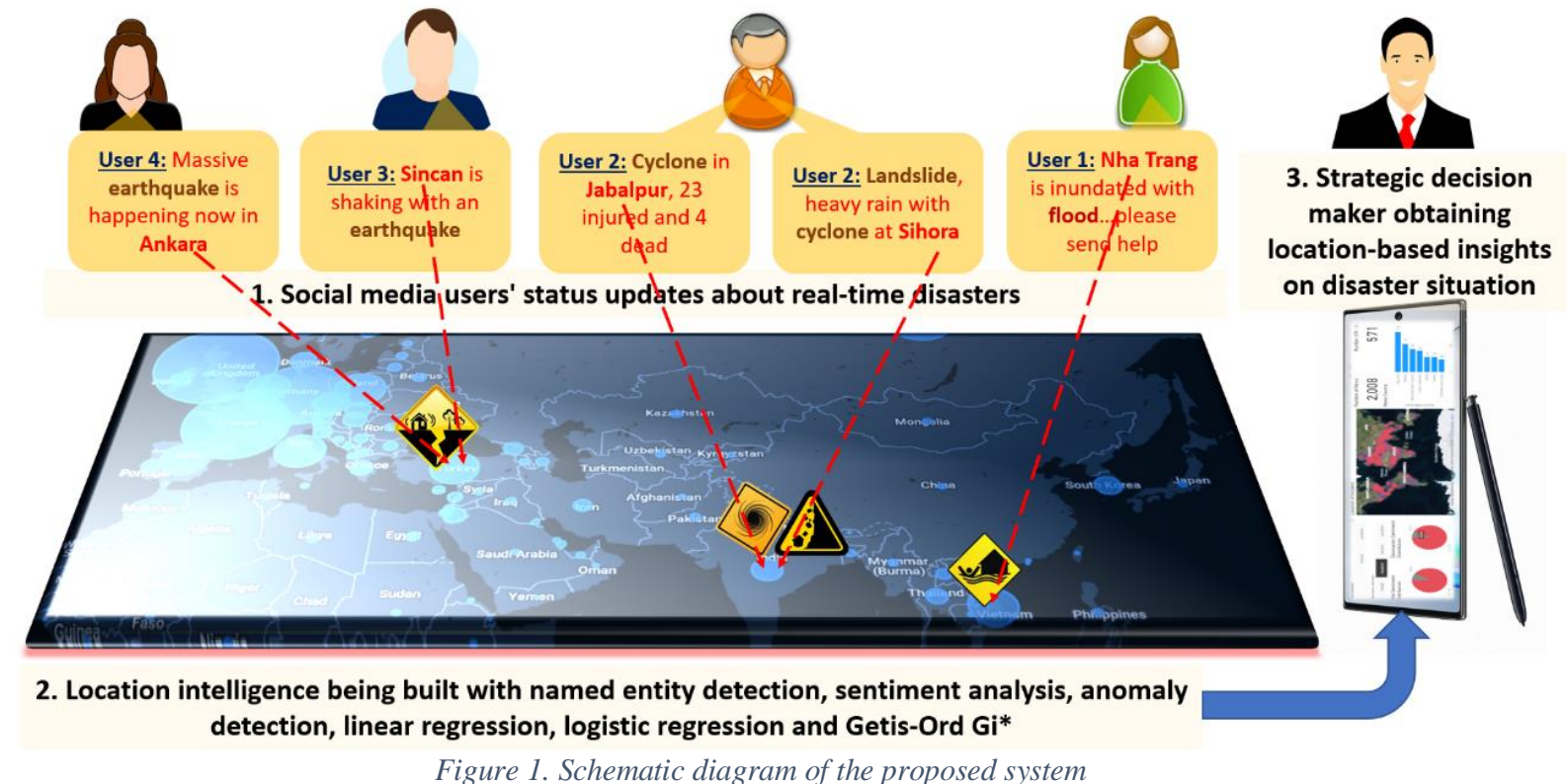

Figure 1. Schematic diagram of the proposed system

social media services like Facebook, Twitter, Instagram that 
5. End-to-End automation making it a sustainable solution with low cost and fast detection

6. Generated 67,528 records of disaster related tweets associated with sentiments, entities, and category classifications, in public database, which would facilitate other researchers to conduct further disaster related studies for benchmarking purpose [19]

Moreover, the data generated through this study adheres to the first element of efficient early warning systems with "systematic collection of data" as mandated by United Nations [5].

According to the literature and the best of our knowledge this is the first study that uses social media sentiment as an inputfeature to CNN based deep learning for extracting disaster location intelligence from social media with the highest level of accuracy (average precision, F1-Score and accuracy were measured to be $0.93,0.90$ and 0.97 respectively).

\section{BACKGROUND}

In [8], earthquake was monitored from Japanese tweets. Within [8], support vector machine (SVM) algorithm was used on tokenized words. Researchers in [9] demonstrated a system that worked only on flood and on an extremely limited set of language (i.e., English, Italian, Portuguese, and Turkish). In [9], word tokenizer and n-gram token were used along with a classifier. The study in [10], demonstrated situational awareness from english tweets. However, researcher in [10], only depicted applicability on earthquake and plane crash disasters. Researchers in [11] investigated Hindi Tweet for only six specific disaster events namely, 1) two bomb blasts in Hyderabad, India; 2) A shooting event that killed 20 children and 6 adults at school in Connecticut, USA; 3) flood and associated landslides in Uttaranchal, India; 4) Typhoon hagupit (i.e., a cyclone) in Philippines; 5) earthquake in Nepal; 6) derailing of train near Harda in India. [11] used Parts of Speech (POS) tagging with classification algorithm. Study in [12] provided a unique perspective in combining dictionary-based sentiment analysis with location information in visualizing theme park visitors' emotions in map. However, [12] only worked on english tweets and used lookup against a dictionary of emotions and utilized algorithms like spatial Autocorrelation, centro-graphic analysis, point pattern analysis, standard deviational ellipse analysis and word frequency analysis. Researchers in [13] used Sentiment Analysis, Naïve Bayes (NB), SVM, and Recurrent Neural Network (RNN) on english tweets to assess users' satisfaction on wireless services. Study in [14], performed sentiment analysis on the highly opinionated political debate in the Citizenship Amendment Act (CAA) using SVM. For this study, they extracted english Tweets from ten different regions of India (i.e., Assam, Andhra Pradesh, Uttar Pradesh, Maharashtra, West Bengal, Tamil Nadu, Kerala, Karnataka, Punjab, and Delhi) [14]. None of these researchers (i.e., [8] [9] [10] [11] [12] [13] [14]) used NER for extracting location from tweets nor they have used the output of sentiment analysis as a predecessor to deep learning methods like CNN. As a result, these studies in suffered poor performance in terms of accuracy on location intelligence [8]
[9] [10] [11] [12] [13] [14].

A recent study in sentiment performed analysis of a largescale dataset consisting of 90,000 COVID-19-related tweets collected in the first two months of the pandemic (February to March 2020) [20]. The study [20] compared the results of analyses using traditional machine learning methods, such as support vector machine, naive Bayes, decision tree, and random forest, and those of deep learning methods, such as CNN, and bidirectional long short-term memory, in combination with various embedding vectors, such as fastText [28], Glove [29], and Word2Vec [30] to classify this COVID-19 tweet dataset into three classes (negative, positive, and neutral). Their results in [20] depict that deep learning-based methods outperform traditional machine learning methods. Hence, usage of deep learning methods is used as one of criteria to assess existing research in social media-based disaster monitoring.

Other than usage of deep learning method, the other criteria for assessing the drawbacks of existing studies in Twitter based disaster monitoring would be "Language coverage", "number of disasters monitored", "using sentiment analysis", "mobile app availability", "type of location service used" (e.g., Geotagged, NER or Lookup) and "data availability for research reusability". Table I shows these criteria for assessing existing literature and how the proposed system compares with the existing studies. Table I clearly shows the drawback of the existing research and how the proposed method aims to provide an AI \& NLP based real-time (as well as fully automated) solution.

TABLE I

EVALUATION OF EXISTING RESEARCH IN TWEETER BASED LOCATION EXTRACTION METHODOLOGY

\begin{tabular}{|c|c|c|c|c|c|c|c|}
\hline 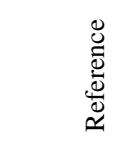 & 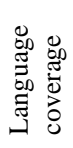 & 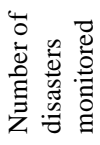 & 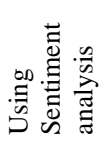 & 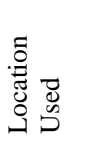 & 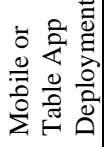 & 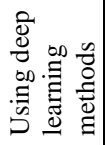 & 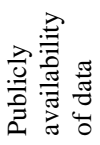 \\
\hline [8] & 1 & 1 & No & GeoTag & No & No & No \\
\hline [9] & 4 & 1 & No & Lookup & No & No & No \\
\hline [10] & 2 & 2 & No & GeoTag & No & No & No \\
\hline [11] & 2 & 6 & No & GeoTag & No & No & No \\
\hline [12] & 1 & 0 & Yes & GeoTag & No & $\mathrm{No}$ & $\mathrm{No}$ \\
\hline [13] & 1 & 0 & Yes & GeoTag & No & No & No \\
\hline [14] & 1 & 0 & Yes & GeoTag & No & No & No \\
\hline Proposed & 110 & 10 & YES & NER & YES & YES & YES \\
\hline
\end{tabular}

\section{METHOD}

As depicted in Fig. 2, live social media feeds like Tweets comes as the input to the pre-possessing stage. Within the preprocessing stage multiple features are extracted. The output of category classification, sentiment analysis and NER process are used as features to train and test the AI models in subsequent stages. CNN based anomaly detection, Getis-Ord $\mathrm{Gi}^{*}$ and automated regression (both linear and logistic) regressions are used within the scope of AI \& Statistical Algorithm. Finally, deep insights are obtained on disaster situation. 
$>$ REPLACE THIS LINE WITH YOUR MANUSCRIPT ID NUMBER (DOUBLE-CLICK HERE TO EDIT) <

Within the Pre-Processing stage, Category classification returns the scalar value of $g_{i}$. Next, Sentiment Analysis step returns four different values for each of the message texts, as a list, $\left\{s_{i}, s_{i}^{\text {Positive }}, s_{i}^{\text {Negative }}, s_{i}^{\text {Neutral }}\right\}$, when $s_{i}$ can take any sentiment category values within \{positive, negative, neutral, mixed . Moreover, $s_{i}^{\text {Positive }}$ or $s_{i}^{\text {Negative }}$ or $s_{i}^{\text {Neutral }}$ will have
Other than assessing customer feedback, category classification has also been used to discover the interest or inquisitiveness of an online social media user [22]. Preprocessing stage uses category classification along with sentiment detection and NER to perform feature attribute extraction for the purpose of further analysis with deep learning Algorithms. Category classification routine could be invoked

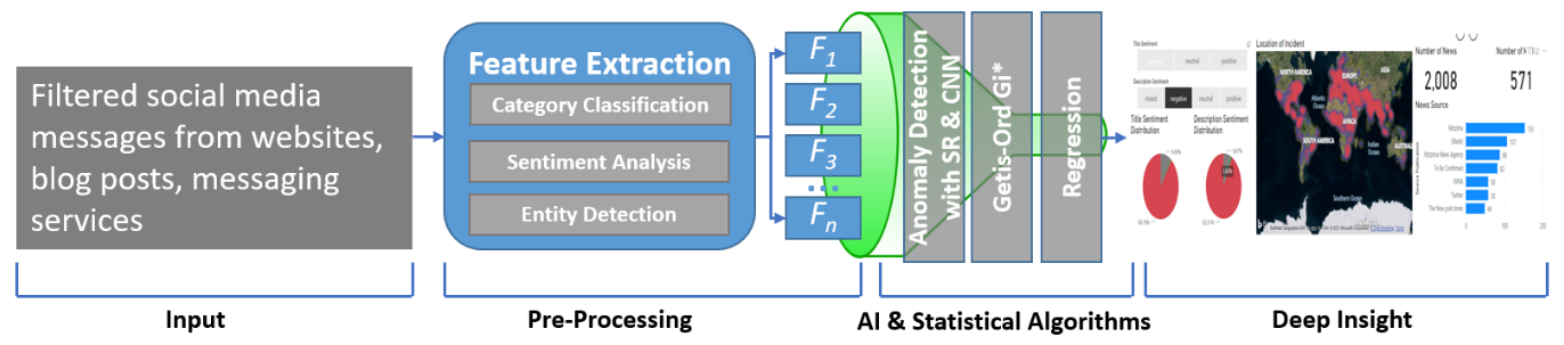

Figure 2. Methodological framework of obtaining real-time automated disaster intelligence

additional values (between 0 and 1 ) representing the confidence levels for each sentiment categories. Finally, NER process returns a list of entities along with their corresponding categories as list of lists, $\left\{\left\{c_{1}, e_{1}^{m_{i}}\right\},\left\{c_{2}, e_{2}^{m_{i}}\right\},\left\{c_{3}, e_{3}^{m_{i}}\right\}, \ldots,\left\{c_{k}, e_{i}^{m_{i}}\right\}\right\}$. Finally, for each of the social media messages, the combined feature is obtained with, $\quad\left\{\left\{\left\{c_{k}, e_{i}^{m_{i}}\right\}\right\},\left\{s_{i}, s_{i}^{\text {Positive }}, s_{i}^{\text {Negative }}, s_{i}^{\text {Neutral }}\right\}, g_{i}\right\}$. Algorithm 1 demonstrates the step-by-step pseudocode for extracting feature attributes within pre-processing stage by using category classification, sentiment analysis, and NER.

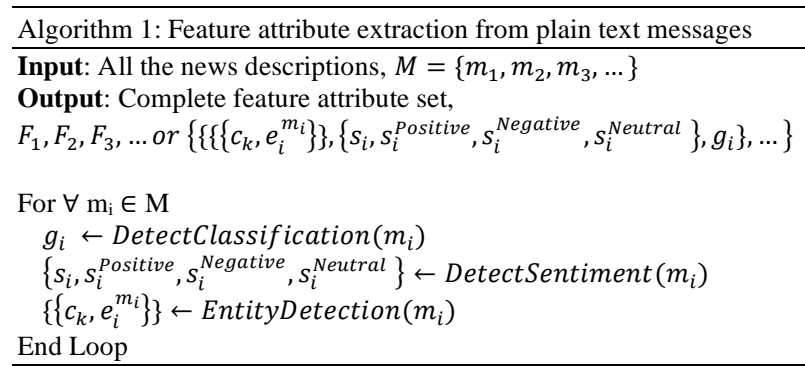

The core concepts behind NLP based category classification, NLP based sentiment analysis, NLP based NER, CNN based anomaly detection, AI based regression and heat map generation with Getis-Ord $\mathrm{Gi}^{*}$ are described in following subsections.

\section{A. Category Classification}

Category classification is applied to classify text inputs into categories that are suitable for a certain business case. There are prebuild models of category classification (e.g., Customer Feedback) available via Microsoft's AI-Builder [21] that can categorize a text input to any of the following categories:

- Issues

- Compliment

- Customer Service

- Documentation

- Price \& Billing

- Staff through MS Power Automate [23] as seen from Fig. 3.

\section{B. Sentiment Analysis}

Research on sentiment analysis began in 2002 with the publication of two studies: [24] and [25]. Research in [24] represented a supervised learning corpus-based machine classifier and [25] exhibited an unsupervised classifier based on linguistic analysis. Previously, the emphasis of sentiment analysis was mostly on product and movie reviews. It spread across other domains with the advent of social media users. Several other studies followed, like studies in [20] [26] [27] [28] [29] [30] [14] [24] [25]. Recent study on Sentiment Analysis has been used to for assessing customer feedback towards comprehending political sentiment of people, specifically to predict election result [31]. All these existing studies in [24] [25] [20] [26] [27] [28] [29] [30] [14] [31] did not employ sentiment analysis as a pre-processor to AI based algorithms with the intent of extracting feature attributes from text inputs. Code 1 shows an example of calling Sentiment Analysis method in C\# using Microsoft Cognitive Services Text Analytics API [32]. Output 1 shows the result of sentiment analysis, where the first sentence was detected as positive, and the second sentence was detected to be neutral in nature.

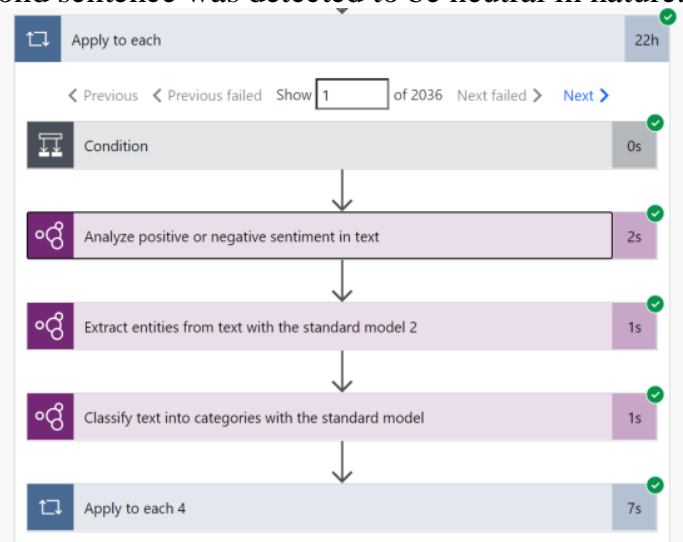

Figure 3. Implementation and evaluation of AI-Feature with Microsoft Power Automate 
$>$ REPLACE THIS LINE WITH YOUR MANUSCRIPT ID NUMBER (DOUBLE-CLICK HERE TO EDIT) <

Other than invoking the API through C\# in .Net environment, sentiment analysis method could also be invoked through MS Power Automate [23] as seen from Fig. 3.

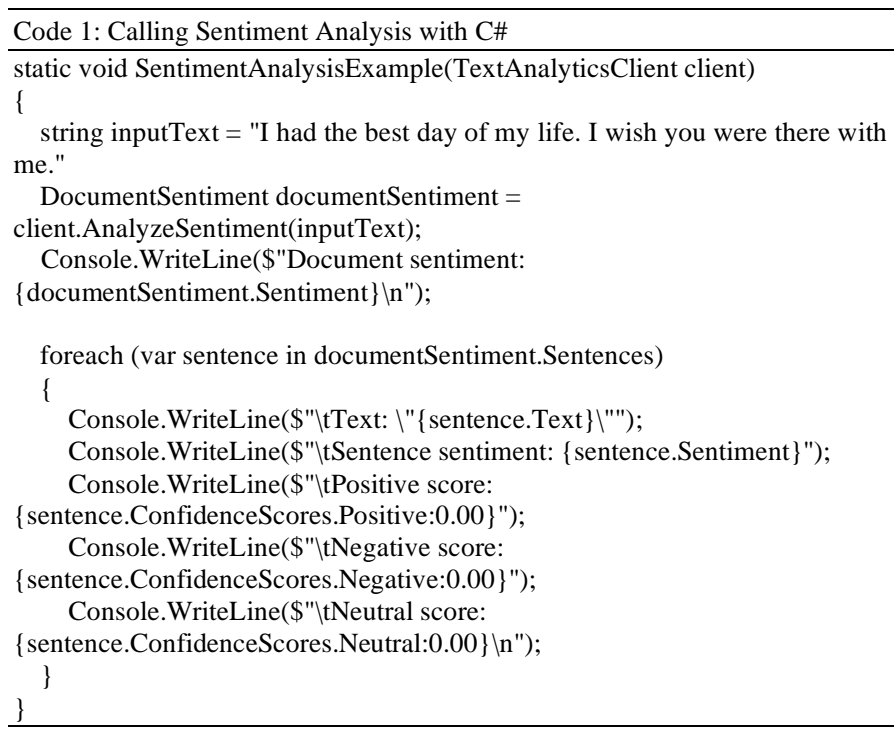

Console Output 1: Sentiment Analysis

Document sentiment: Positive

Text: "I had the best day of my life."

Sentence sentiment: Positive

Positive score: 1.00

Negative score: 0.00

Neutral score: 0.00

Text: "I wish you were there with me."

Sentence sentiment: Neutral

Positive score: 0.21

Negative score: 0.02

Neutral score: 0.77

\section{Named Entity Recognition (NER)}

NER is an NLP based information extraction task that seeks to locate and classify named entities mentioned in unstructured text into pre-defined categories such as person names, organizations, locations, date / time expressions, quantities, monetary values, numeric values, percentages, etc. NER has been applied in almost all areas to extract crucial information from unstructured texts [33], [27]. Previous research has extracted three different categories of entities (i.e., "Disease or Syndrome", "Sign or Symptom" and "Pharmacologic Substance") from health-related tweet messages [33] for discovering public health information and developing just-intime disease outbreak prediction system and drug interactions system. In [27], Basic NLP based methodologies are applied to extract entities and relationships, and to identify sentiment. The keywords investigated within [27] were Drug Abuse Cannabinoids, Buprenorphine, Opioids, Sedatives and Stimulants. Research in [28], qualitatively evaluated posts about methylphenidate from five French patient web-forums including an analysis of information about misuse or abuse. Data were accumulated from French social networks that cited methylphenidate keywords. Text mining methods such as NER and topic modeling were used to analyze the chatter, including the identification of adverse reactions. Previous research in
[33], [27], [28] did not use NER as a pre-processor for AI based algorithms. Code 2 shows an example of invoking NER with C\# using Microsoft Cognitive Services Text Analytics application programming interface (API) [32]. Console Output 2 shows the result of calling NER. As we can observe from the example "trip" was successfully classified at an event, "Seattle" was classified as a location, "last week" was classified as DateTime category.

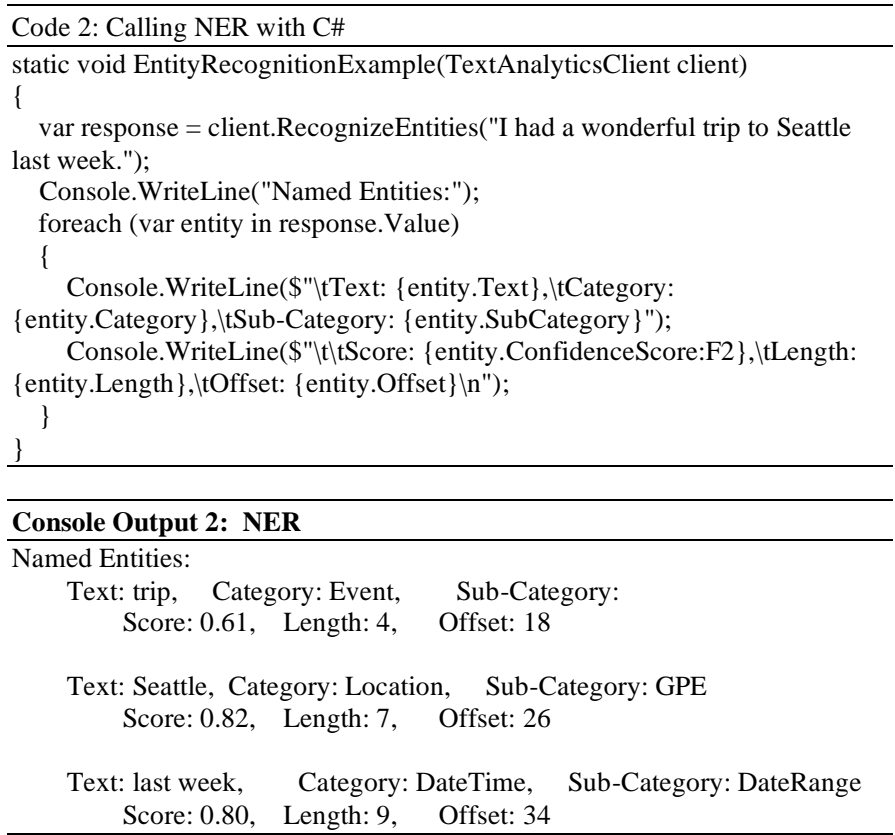

Other than calling the API through C\# in .Net environment, NER method could also be invoked through MS Power Automate [23] as seen from Fig. 3.

\section{Anomaly Detection}

The anomaly detection process augments line charts used within MS Power BI by automatically detecting anomalies within time-series data. It also provides NLP based explanations on anomalies to assist root-cause analysis. In our most recent study, we have used the anomaly detection process for detecting abnormal cases of landslides and acquired the root causes of these anomalies [6] [7]. We have also used CNN based Anomaly detection for accurately identifying abnormalities in global events [15]. Before assessing the details of anomaly detection, problem definition must be presented.

Problem 1: Given a sequence of real values, $x=$ $x_{1}, x_{2}, x_{3}, \ldots, x_{n}$, the task of time-series anomaly detection is to produce an output sequence $y=y_{1}, y_{2}, y, \ldots, y_{n}$, where $y_{i} \in$ $\{0,1\}$ indicates whether $x_{i}$ is an anomaly point.

The implemented solution used the Spectral Residual (SR) from the visual saliency detection domain and then applied a CNN to the results generated by the SR model [34]. The SR algorithm encompasses the following three major steps:

1. Perform Fourier transform to obtain the log amplitude spectrum.

2. Determine the SR.

3. Perform inverse Fourier transform that converts the sequence back to the spatial domain. 
$>$ REPLACE THIS LINE WITH YOUR MANUSCRIPT ID NUMBER (DOUBLE-CLICK HERE TO EDIT) <

$$
\begin{aligned}
& A(f)=\text { Amplitude }(f(x)) \\
& P(f)=\operatorname{Phrase}(f(x)) \\
& L(f)=\log (A(f)) \\
& A L(f)=h_{q}(f) \cdot L(f) \\
& R(f)=L(f)-A L(f) \\
& S(x)=\left\|f^{-1}(\exp (R(f)+i P(f)))\right\|
\end{aligned}
$$

where $f$ and $\mathrm{f}^{1}$ denote the Fourier transform and inverse Fourier transform, respectively; $\mathrm{x}$ is the input sequence with shape $\mathrm{nX} 1 ; \mathrm{A}(\mathrm{f})$ is the amplitude spectrum of sequence $\mathbf{x} ; \mathrm{P}(\mathrm{f})$ is the corresponding phase spectrum of sequence $\mathrm{x} ; \mathrm{L}(\mathrm{f})$ is the $\log$ representation of $\mathrm{A}(\mathrm{f})$; and $\mathrm{AL}(\mathrm{f})$ is the average spectrum of $\mathrm{L}(\mathrm{f})$, which can be approximated by convoluting the input sequence by $h_{q}(f)$, where $h_{q}(f)$ is a $q \times q$ matrix noted as:

$h_{q}(f)=\frac{1}{q^{2}}\left[\begin{array}{ccc}1 & 1 & \ldots 1 \\ 1 & 1 & \ldots 1 \\ \ldots & \vdots & \ddots \\ 1 & 1 \ldots & 1\end{array}\right]$

$R(f)$ is the SR, which is the $\log$ spectrum $L(f)$ minus the averaged $\log$ spectrum $\mathrm{AL}(\mathrm{f})$. The SR serves as a condensed representation of the sequence, whereas the novelty part of the original sequence becomes more significant. Last, the sequence was transferred back to the spatial domain using an inverse Fourier transform. The resultant sequence $\mathbf{S}(\mathbf{x})$ is described to as the saliency map [35]. The values of the anomaly points are determined as follows:

$x=(\bar{x}+$ mean $)(1+$ var $) \cdot r+x$

where $\bar{x}$ is the local average of the preceding points, mean and var are the mean and variance of all points within the current sliding window, and $r \sim \mathrm{N}(0,1)$ is randomly sampled. In this process, $\mathrm{CNN}$ is applied to the saliency map instead of to the raw input, thus improving the efficiency of the overall process of anomaly detection [34] [35].

The anomaly detection algorithm delivers explanations for all detected anomalies following the root-cause analysis performed by the AI \& NLP services. In fact, our implementation of anomaly detection was performed in three steps:

1. Detect all the anomalies within the time series (i.e., any values that reside outside the threshold range).

2. Identify the key drivers of these anomalies.

3. Describe the results in a natural language (explanation of the root cause) using NLP [18].

\section{E. Heat map generation}

The heat map generation process detected locality associated with high level and low-level disaster related tweets. Getis-Ord $\mathrm{Gi}^{*}$ statistic is the method used to identify statistically significant spatial clusters at local scale [36]. The final outputs offered by the Heat Map Algorithm were the significant hotspot and cool-spot areas, spatially defined by Eq. (9) to Eq. (11). The Getis-Ord local statistic is given as:

$G_{i}^{*}=\frac{\sum_{j=1}^{n} w_{i, j} x_{j}-\bar{X} \sum_{j=1}^{n} w_{i, j}}{\sqrt{\frac{n \sum_{j=1}^{n} w_{i, j}^{2}-\left(\sum_{j=1}^{n} w_{i, j}\right)^{2}}{n-1}}}$

where $x_{j}$ is the attribute value for feature $j, w_{i, j}$ is the spatial weight between feature $i$ and $j, n$ is equal to the total number of features and:

$\bar{X}=\frac{\sum_{j=1}^{n} x_{j}}{n}$
$S=\sqrt{\frac{\sum_{j=1}^{n} x_{j}^{2}}{n}-(\bar{X})^{2}}$

The $\mathrm{G}_{\mathrm{i}}{ }^{*}$ statistic is a z-score and hence further calculations are not mandated. GenerateHeatmap() function of Algorithm 2 implements Eq. (9) to Eq. (11) for the location extraction process. GeoDecode() function within Algorithm 2 extracts the longitude and latitude of a location.

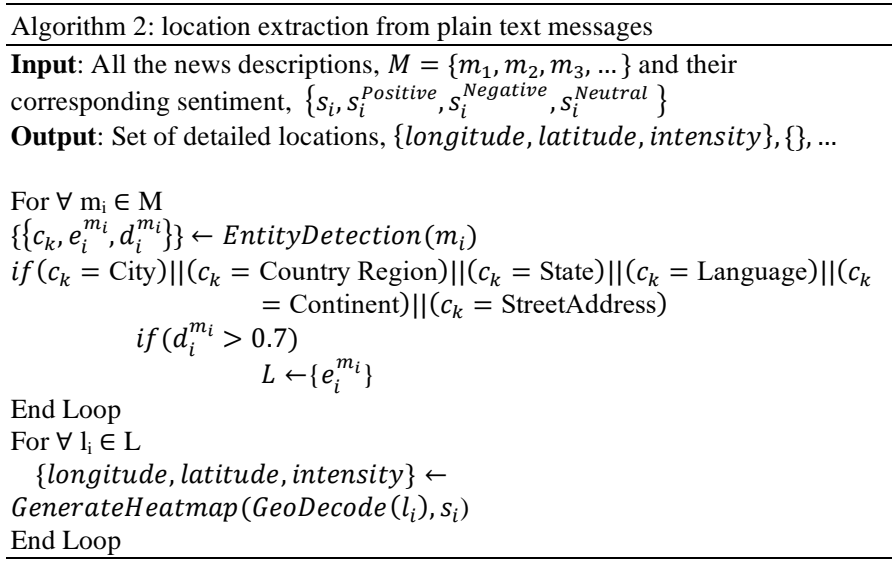

\section{F. Regression Analysis}

The proposed solution uses two different types of regression analysis (i.e., linear and logistic regression) with MS Power BI's Key influencers visual to find the relationship between disaster feature attributes and the level of negativity of social media messages. For numerical features, linear regression was implemented using Microsoft's ML.Net's SDCA regression implementation [37]. The aim of linear regression is to obtain the best-fit line that can accurately predict the output for the continuous dependent variable. By discovering the best-fit line, the algorithm proves a linear relationship between the dependent and independent variables in the form of Eq. (12). $y=b_{0}+b_{1} x_{1}+\varepsilon$

In contrast, for categorical features, logistic regression was implemented using ML.Net's L-BFGS logistic regression [38] [39]. Logistic regression is one of the most prevalent Machine Learning (ML) algorithms that use supervised learning techniques. Logistic regression was applied to predict the categorical dependent variable with the help of independent variables using Eq. (13).

$\log [y / y-1]=b_{0}+b_{1} x_{1}+b_{2} x_{2}+\cdots b_{n} x_{n}$

\section{RESULT}

We used Microsoft Power Automate and Microsoft Power Query with $M$ Language to automatically retrieve real-time twitter feeds. Within the Microsoft Power Automate pipeline NER, sentiment analysis and category classification were performed. These results were finally stored within Microsoft SQL Server at the end of the process. Subsequently Microsoft Power BI was used to display, analyze, and visualize the results.

The real-time automated system was tested and evaluated from 28 Sep. 2021 till 6 Oct. 2021. 67,528 Tweets with 67515 entities of 39 different languages were processed during this period. The NER classified 67515 entities into 27 different types of entity categories as seen from Table II. 
TABLE II

ENTITY CATEGORIES WITH THE NUMBER OF ENTITIES

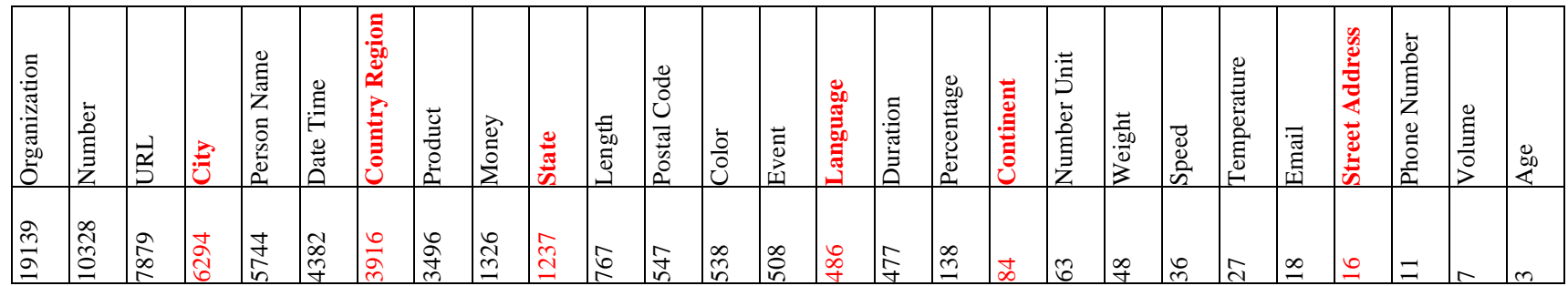

Out of these 27 entity categories City, Country Region, State, Language, Continent and Street Address contains location related entities. These 6 location related entities are highlighted in Table II.

As seen earlier, in Algorithm 2, the entity extraction function generates 3 outputs, namely, entity category $c_{k}$, entity $e_{i}^{m_{i}}$, and entity confidence $d_{i}^{m_{i}}$. Only entities extracted with more than $70 \%$ confidence are subsequently processed by the location extraction algorithm (i.e., Algorithm 2). Hence, the count of extracted location entities differs in Table III from Table II, as Table III only shows location entities greater than $70 \%$ confidence level.

TABLE III

9727 LOCATION ENTITIES WITH GREATER THAN 70\% CONFIDENCE EXTRACTED BY LOCATION EXTRACTION Algorithm (I.E. Algorithm 2)

\begin{tabular}{|l|r|l|l|l|l|}
\hline & $\begin{array}{l}\text { Count } \\
\text { of } \\
\text { Entity }\end{array}$ & $\begin{array}{l}\text { Negative } \\
\text { Sentiment } \\
\text { Confidence }\end{array}$ & $\begin{array}{l}\text { Positive } \\
\text { Sentiment } \\
\text { Confidence }\end{array}$ & $\begin{array}{l}\text { Neutral } \\
\text { Sentiment } \\
\text { Confidence }\end{array}$ & $\begin{array}{l}\text { Number of } \\
\text { Disaster } \\
\text { Categories }\end{array}$ \\
\hline City & 5751 & 0.46 & 0.22 & 0.32 & 10 \\
\hline Country Region & 2905 & 0.67 & 0.07 & 0.26 & 10 \\
\hline State & 489 & 0.55 & 0.10 & 0.35 & 10 \\
\hline Language & 486 & 0.14 & 0.08 & 0.78 & 10 \\
\hline Continent & 80 & 0.19 & 0.06 & 0.75 & 8 \\
\hline Street Address & 16 & 0.413 & 0.29 & 0.30 & 5 \\
\hline
\end{tabular}

Table IV shows the extracted location entities by each of the disaster categories. 10 different types of disasters were successfully identified during the monitored period. As shown in Table IV, earthquake and tsunami had the highest of numbers of tweets. People reacted more for Tsunami, bushfire, and Landslide events through favorite and retweets.

As shown in Table V, all 67,528 tweets were categorized by the category classification method in 6 different categories: issues, compliment, staff, documentation, price \& billing, customer service. Out of the 39 languages English had the greatest number of tweets with 54342 tweets, Japanese had the second highest with 7383 tweets followed by Spanish with 1177 tweets. The least number of tweets were captured for Bulgarian, Finnish and Telugu languages.

Fig. 4 shows the heatmap generated by algorithm 2, which shows the disaster locations extracted in Realtime from monitored twitter feeds. Using these heatmaps a decision maker obtains Realtime updates about various types of disasters with high level of confidence (as algorithm 2 only extracts location related entities with more than $70 \%$ confidence).
TABLE IV

ANALYSIS OF DISASTER SENTIMENT WITH LOCATION ENTITIES

\begin{tabular}{|c|c|c|c|c|c|c|c|}
\hline $\begin{array}{l}\ddot{0} \\
\ddot{\Xi} \\
\ddot{D}\end{array}$ & 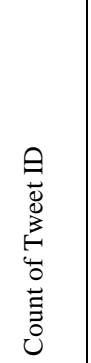 & 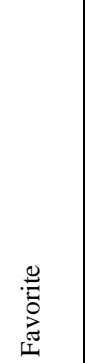 & $\begin{array}{l}\overrightarrow{0} \\
\stackrel{0}{0} \\
\stackrel{0}{\alpha}\end{array}$ & 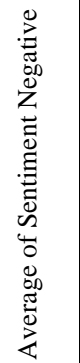 & 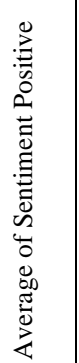 & 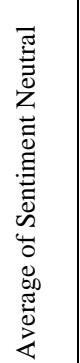 & 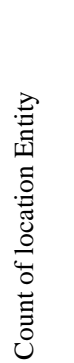 \\
\hline Bushfire & 6630 & 13563 & 3932 & 0.53 & 0.159 & 0.328 & 530 \\
\hline \begin{tabular}{|l|} 
Cyclone \\
\end{tabular} & 5829 & 4127 & 1057 & 0.34 & 0.18 & 0.48 & 731 \\
\hline Drought & 4278 & 4514 & 1763 & 0.66 & 0.14 & 0.20 & 404 \\
\hline Earthquake & 12226 & 3373 & 1014 & 0.347 & 0.27 & 0.38 & 2142 \\
\hline \begin{tabular}{|l|} 
Flood \\
\end{tabular} & 2250 & 456 & 253 & 0.55 & 0.102 & 0.35 & 276 \\
\hline Hurricane & 7234 & 2863 & 1076 & 0.641 & 0.10 & 0.26 & 959 \\
\hline Landfall & 6123 & 10794 & 3607 & 0.52 & 0.07 & 0.41 & 1077 \\
\hline Landslide & 8818 & 12864 & 4890 & 0.61 & 0.10 & 0.29 & 1956 \\
\hline Tsunami & 11988 & 18625 & 1262 & 0.42 & 0.17 & 0.41 & 696 \\
\hline Volcano & 2152 & 6789 & 524 & 0.41 & 0.22 & 0.36 & 467 \\
\hline
\end{tabular}

Fig. 5 demonstrates CNN based anomaly detection process working live in following 3 steps:

1. Identifying all the anomalies in terms of negativity of the message

2. Identifying possible root causes of all anomalies

3. Describe the anomaly along with all the root causes in plain english language using NLP

As seen from Fig. 5, a disaster strategist clicked on a particular anomaly on $30^{\text {th }}$ Sep 2021. Using the NLP, the anomaly detection process describes the anomaly in plain language as "Negative sentiment was unexpectedly high on 9/30/2021 5:31:00 PM. It had a value of 72.54, which is above the expected range of $43.94-44.91$ ". Moreover, the anomaly detection process describes the root cause of this selected anomaly. For example, for this anomaly one of the root-cause was reply tweet was unusually high, meaning a lot of people replied on this disaster related tweet. Anomaly detection process was $82 \%$ confident on this root-cause. It should be noted that NLP based explanations for the root causes are also presented by our solution, as seen from Fig 5 . 
$>$ REPLACE THIS LINE WITH YOUR MANUSCRIPT ID NUMBER (DOUBLE-CLICK HERE TO EDIT) >

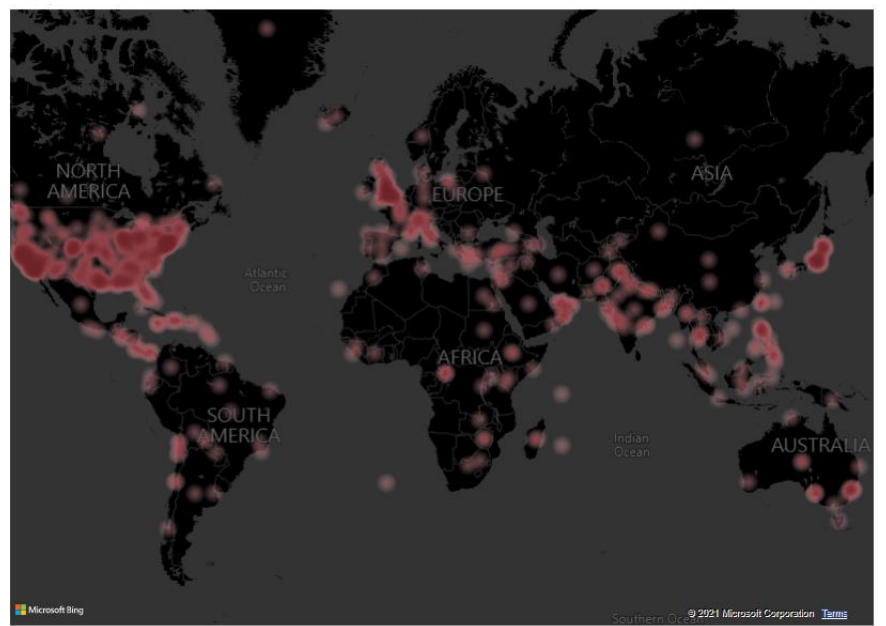

Figure 4. Heat map generated by the proposed system representing the disaster area during the monitored period

NLP is also utilized on explaining insights generated by regression algorithms (both linear and logistic). As shown in Fig. 6, our regression algorithm discovered the correlation between negativity levels of tweet and several other conditions like when tweet language is english, entity type is color (since many tweets mention "code red" to highlight the severity of a disaster), disaster type is drought, disaster category is hurricane, disaster category is landslide, and tweet type is retweet (signifying higher level of interaction).

TABLE V

NUMBER OF TWEETS FOR EACH CATEGORIES

\begin{tabular}{|l|r|}
\hline Category & Count of Tweet ID \\
\hline Issues & 52209 \\
\hline Compliment & 10649 \\
\hline Staff & 2654 \\
\hline Documentation & 1374 \\
\hline Price \& Billing & 628 \\
\hline Customer Service & 14 \\
\hline
\end{tabular}

Moreover, Fig. 6 shows in natural language "When Language is en", the average of negative sentiment increases by 0.3 using NLP.

Based on the result of logistic regression and linear regression, 4 clusters of disasters were discovered as shown in Fig. 7. NLP also describes the first cluster (i.e., segment 1 or the segment with highest negativity) the user highlighted where language is english, disaster type is hurricane and tweet type is retweet (as shown in Fig. 7).

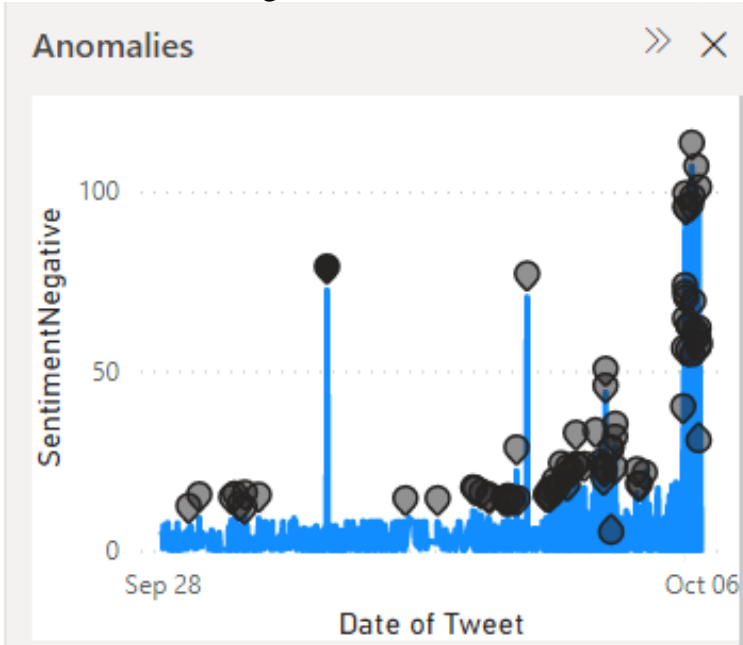

SentimentNegative was unexpectedly high on 9/30/2021 5:31:00 PM. It had a value of 72.54, which is above the expected range of 43.94 44.91.

\section{Possible explanations} Strength (i)

'TweetType' is Reply

SentimentNegative for TweetType Reply was unusually high, which may have lifted the SentimentNegative total.

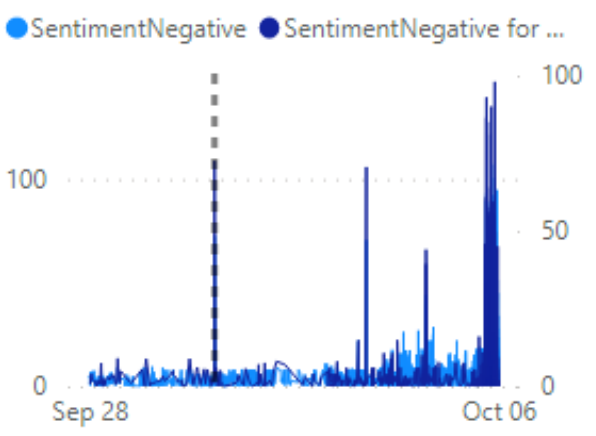

Add to report

Figure 5. CNN based anomaly detection algorithm picked up an abnormally high number of tweets on a particular day, since there were extremely high number of user engagements (with replies) 
$>$ REPLACE THIS LINE WITH YOUR MANUSCRIPT ID NUMBER (DOUBLE-CLICK HERE TO EDIT) <

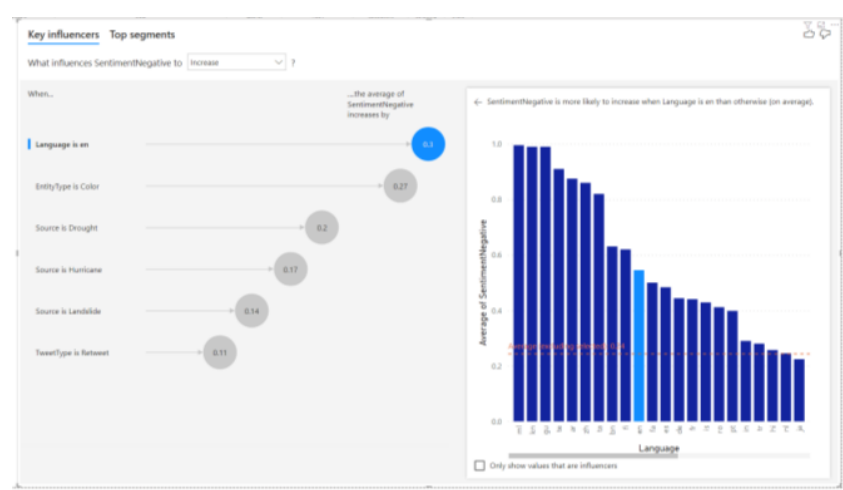

Figure 6. Logistic regression automatically discovered the factors influencing the negativity of disaster related tweets

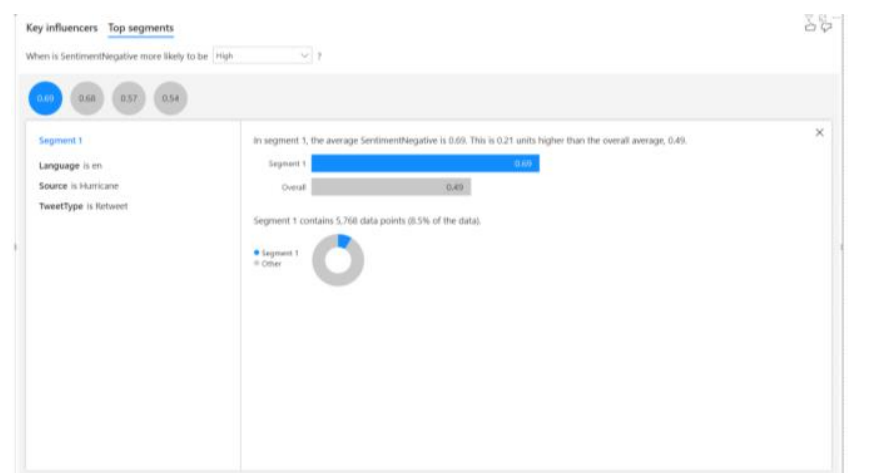

Figure 7. Automated AI based clustering reveals that out of the 4 clusters, English tweets related to Hurricane that has been retweeted caused the highest negativity among the social media users

\section{DISCUSSION}

The usual users of this proposed solution are strategic planners, policymakers, and diplomatic strategists who are concerned with global disasters and their subtle impact on society, and locations (applicable for more than 110 countries). This system would allow users to comprehend the characteristics of a disaster in a particular area and provide useful guidance for policy implementation and diplomatic ties with affected groups, governments, and non-government bodies. Since a typical decision maker requires the information to be presented on an extensive range of devices to support instant decision-making, the deployed solution has been made accessible on a range of devices, including web, tablet, and mobile platforms (i.e., iOS, Android and Windows). As shown in Fig. 8, a user is monitoring the possible disasters in a global map with deep insights (e.g., intensity of the disaster, level of negativity by the social media users etc.) in Samsung Note 10 Lite within Android app. In Fig. 9, a user is assessing the influence of Hurricane on negative user perception. Fig 9 is depicting a tablet deployment of the proposed solution in Samsung A7 Tab.

All modules employed within the presented system were meticulously assessed in terms of True Positives (TP), True Negatives (TN), False Positives (FP), False Negatives (FN), Recall, Precision, F1-Score and Accuracy.

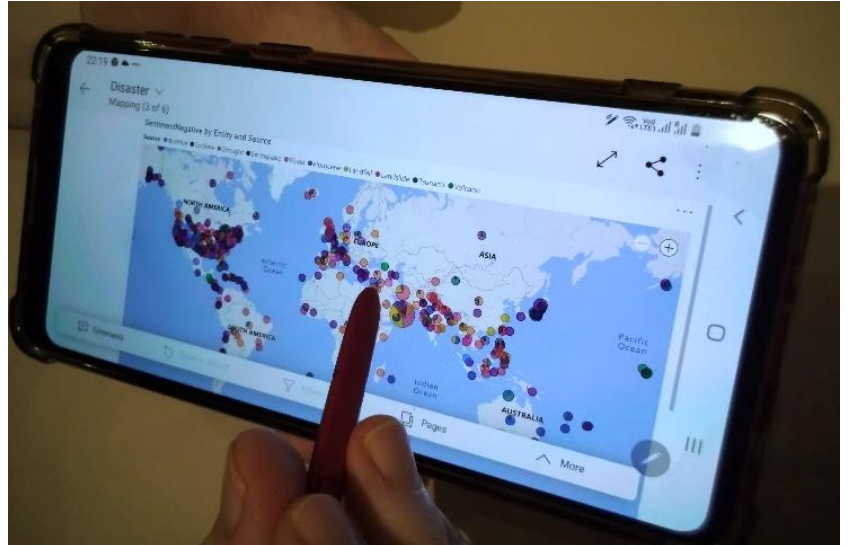

Figure 8. Disaster monitoring solution running in Samsung Note 10 Lite Mobile

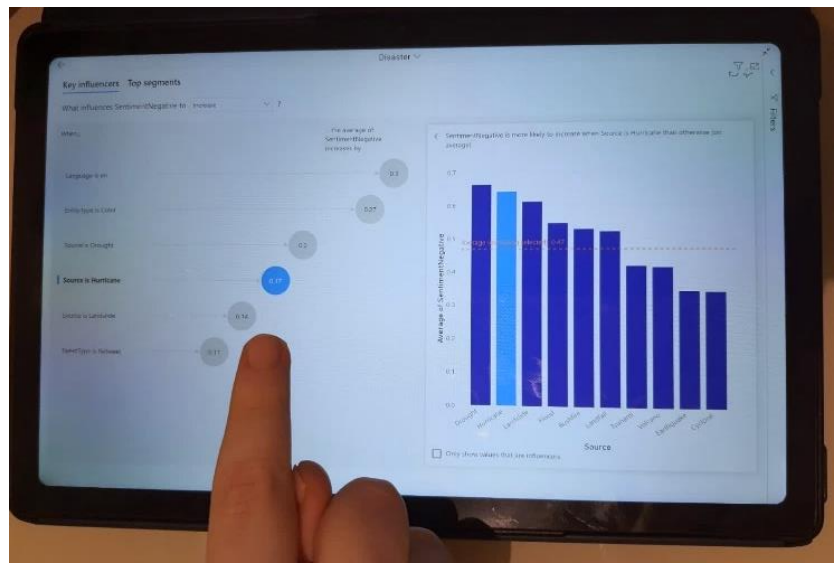

Figure 9. Disaster monitoring solution running in Samsung A7 Tablet

Precision informs what fraction of predictions as a positive class were positive (shown in Eq. (14)).

Precision $=\frac{T P}{T P+F P}$

Recall informs what fraction of all positive samples were accurately predicted as positive by the classifier as shown in Eq. (15). It is also known as True Positive Rate (TPR), Sensitivity, and Probability of Detection.

Recall $=\frac{T P}{T P+F N}$

F1-score unites precision and recall into a single measure as shown in Eq. (16). Mathematically it is the harmonic mean of precision and recall.

$F_{1}$ Score $=\frac{2 X \text { Precision } X \text { Recall }}{\text { Precision }+ \text { Recall }}=\frac{2 X T P}{2 X T P+F P+F N}$

Lastly, Accuracy provides the overall accuracy of the model, and it is represented by the fraction of the total samples that were correctly classified the by the classifier, as seen in Eq. (17).

Accuracy $=\frac{T P+T N}{T P+T N+F P+F N}$

The detailed results with all the evaluation metrics used are presented in Table VI. As seen from Table VI, the highest level of precision was obtained for Cyclone with a precision of 0.96 . Highest level of recall was recorded for earthquake with a recall rate of 0.94. In terms of accuracy bushfire, cyclone, earthquake, and hurricane demonstrated the best performance during our 
$>$ REPLACE THIS LINE WITH YOUR MANUSCRIPT ID NUMBER (DOUBLE-CLICK HERE TO EDIT) <

evaluation. In should be noted that previous research had much lower performance compared with our result (e.g., 0.81 precision in [9]).

\section{CONCLUSION}

Existing research in disaster monitoring through social media is inefficient in following:

- Inaccuracy in classifying a disaster related tweet

- Limited support of language

- Limited support of disaster types

- Inaccuracy in identifying disaster location

- Not supporting disaster insight in plain language with NLP

- $\quad$ Limited support disseminating the insights (i.e., not available in mobile app through iOS or Android)

\section{TABLE VI}

EVALUATION OF THE AUTOMATED DISASTER MONITORING SYSTEM WITH LOCATION INTELLIGENCE

\begin{tabular}{|l|r|r|r|r|r|r|r|r|}
\hline & & & & & & & F1- & \\
& TP & TN & FP & FN & Precision & Recall & Score & Accuracy \\
\hline Bushfire & 499 & 6000 & 31 & 99 & 0.94 & 0.83 & 0.88 & 0.98 \\
\hline Cyclone & 701 & 5001 & 30 & 97 & 0.96 & 0.88 & 0.92 & 0.98 \\
\hline Drought & 378 & 3768 & 26 & 106 & 0.94 & 0.78 & 0.85 & 0.97 \\
\hline Earthquake & 1990 & 9961 & 152 & 123 & 0.93 & 0.94 & 0.93 & 0.98 \\
\hline Flood & 218 & 1900 & 58 & 74 & 0.79 & 0.75 & 0.77 & 0.94 \\
\hline Hurricane & 906 & 6165 & 53 & 110 & 0.94 & 0.89 & 0.91 & 0.98 \\
\hline Landfall & 994 & 4921 & 83 & 125 & 0.92 & 0.89 & 0.9 & 0.97 \\
\hline Landslide & 1821 & 6718 & 135 & 144 & 0.93 & 0.93 & 0.93 & 0.97 \\
\hline Tsunami & 640 & 11033 & 56 & 259 & 0.92 & 0.71 & 0.8 & 0.97 \\
\hline Volcano & 403 & 1607 & 64 & 78 & 0.86 & 0.84 & 0.85 & 0.93 \\
\hline Overall & 8550 & 57074 & 688 & 1215 & 0.93 & 0.88 & 0.9 & 0.97 \\
\hline
\end{tabular}

In this study, we addressed all these limitations with a novel methodology of using social media sentiment as an input feature to CNN based deep learning and by deploying through Microsoft Power Platform ecosystem [7] [6] [15]. Our experimentation with 67528 tweets, entities and sentiments shows that our proposed method can pinpoint a disaster location with 0.93 precision, 0.88 recall, 0.90 F1-Score and 0.97 accuracy.

Using the presented solution, a disaster strategist working in an emergency or crisis management organization can obtain valuable insight harnessing the power of AI and NLP through a range of devices including mobile or tablet (through both iOS and Android Apps).

In future, we would like to merge disaster data obtained through social media along with historical event datasets (e.g., NASA's global landslide inventory [40]) to obtain a more comprehensive understanding of analytical intelligence through deep learning.

\section{ACKNOWLEDGMENT}

The Author would like to thank the Professor Edris Alam of Integrated Emergency Management and Business Continuity Program, Rabdan Academy (UAE) for his valuable input in developing integrated disaster reporting solution presented within this study. Moreover, the Author would like to extend sincere appreciation to Taufiqur Rahman, Development Expert of Federal Government, Australia for his valuable suggestions throughout the implementation of this solution in multiple platforms.

\section{REFERENCES}

[1] Y. W. Rabby and Y. Li, "Landslide Inventory (2001-2017) of Chittagong Hilly Areas, Bangladesh," Data, vol. 5, no. 4, 2020.

[2] Statista, "Cost of natural disaster losses worldwide from 2000 to 2020, by type of loss(in billion U.S. dollars)," 20 December 2021. [Online]. Available:

https://www.statista.com/statistics/612561/natural-disaster-lossescost-worldwide-by-type-of-loss/. [Accessed 21 Dec 2021].

[3] National Centers for Environmental Information, "Billion-Dollar Weather and Climate Disasters," [Online]. Available: https://www.ncdc.noaa.gov/billions/events/US/1980-2021. [Accessed 20 December 2021].

[4] World Bank, "Economics for Disaster Prevention and Preparedness in Europe," 4 June 2021. [Online]. Available: https://www.worldbank.org/en/news/feature/2021/06/04/economic s-for-disaster-prevention-and-preparedness-in-europe. [Accessed 2 December 2021].

[5] United Nations, "Early Warning Systems," [Online]. Available: https://www.un-spider.org/risks-and-disasters/early-warningsystems\#no-back. [Accessed 3011 2021].

[6] F. K. Sufi and M. Alsulami, "Knowledge Discovery of Global Landslides Using Automated Machine Learning Algorithms," IEEE Access, vol. 9, 2021.

[7] F. K. Sufi, "Al-Landslide: Software for acquiring hidden insights from global landslide data using Artificial Intelligence," Software Impacts, vol. 10, no. 100177, 2021.

[8] T. Sakaki, M. Okazaki and Y. Matsuo, "Tweet Analysis for Real-Time Event Detection and Earthquake Reporting System Development," IEEE Transactions on Knowledge and Data Engineering, vol. 25, no. 4, pp. 919-931, 2013.

[9] S. E. Middleton, L. Middleton and S. Modafferi, "Real-time crisis mapping of natural disasters using social media," IEEE Intelligent Systems, vol. 29, pp. 9-17, 2014.

[10] J. Yin, A. Lampert, M. Cameron, B. Robinson and R. Power, "Using Social Media to Enhance Emergency Situation Awareness," IEEE Intelligent Systems, vol. 27, no. 6, pp. 52-59, 2012.

[11] K. Rudra, N. Ganguly, P. Goyal and S. Ghosh, "Extracting and Summarizing Situational Information from the Twitter Social Media during Disasters," ACM Transactions on the Web, vol. 12, no. 3, pp. 17 (1-35), 2018.

[12] S. B. Park, J. Kim, Y. K. Lee and C. M. Ok, "Visualizing theme park visitors' emotions using social media analytics and geospatial analytics," Tourism Management, vol. 80, no. 104127, 2020. 


\section{$>$ REPLACE THIS LINE WITH YOUR MANUSCRIPT ID NUMBER (DOUBLE-CLICK HERE TO EDIT) <}

[13] W. Qi, R. Procter, J. Zhang and W. Guo, "Mapping Consumer Sentiment Toward Wireless Services Using Geospatial Twitter Data," IEEE Access, vol. 7, pp. 113726-113739, 2019.

[14] G. Vashisht and Y. N. Sinha, "Sentimental study of CAA by locationbased tweets," International Journal of Information Technology, 2021.

[15] F. K. Sufi and M. Alsulami, "Automated Multidimensional Analysis of Global Events With Entity Detection, Sentiment Analysis and Anomaly Detection," IEEE Access, vol. 9, pp. 152449 - 152460, 2021.

[16] F. K. Sufi, "Al-GlobalEvents: A Software for analyzing, identifying and explaining global events with Artificial Intelligence," Software Impacts, vol. Accepted In Press, 2021.

[17] T. B. N. Hoang and J. Mothe, "Location extraction from tweets," Information Processing \& Management, vol. 54, no. 2, pp. 129-144, 2018.

[18] Microsoft Documentation, "Choosing a Natural language processing technology in Azure," [Online]. Available: Microsoft. https://docs.microsoft.com/en-us/azure/architecture/dataguide/technology-choices/natural-language-processing . [Accessed 21 August 2021].

[19] F. K. Sufi, "Analyzing Natural Disaster related Tweets with Al and NLP based Services," IEEE Dataport, doi: https://dx.doi.org/10.21227/p0ed-cb23, 2021.

[20] U. Naseem, I. Razzak, M. Khushi, P. W. Eklund and J. Kim, "COVIDSenti: A Large-Scale Benchmark Twitter," IEEE TRANSACTIONS ON COMPUTATIONAL SOCIAL SYSTEMS, 2020.

[21] M. Documentation, "Category Classification Model," 2021. [Online]. Available: https://docs.microsoft.com/en-us/ai-builder/prebuiltcategory-classification. [Accessed 1 Oct 2021].

[22] T. Hong, J.-A. Choi, K. Lim and P. Kim, "Enhancing Personalized Ads Using Interest Category Classification of SNS Users Based on Deep Neural Networks," Sensors, vol. 21, no. 199, 2021.

[23] Microsoft Documentation, "Microsoft Power Automate," 2021. [Online]. Available: https://docs.microsoft.com/en-us/powerautomate/. [Accessed 29 August 2021].

[24] B. Pang, L. Lee and S. Vaithyanathan, "Thumbs up?: Sentiment classification using machine learning techniques," in Conf. Empirical Methods Natural Lang. Process., 2002.

[25] P. D. Turney, "Thumbs up or thumbs down?: Semantic orientation applied," in 40th Annu. Meeting, 2002.

[26] L. Li, Q. Zhang, X. Wang and J. Zhang, "Characterizing the Propagation of Situational Information in Social Media During COVID-19 Epidemic: A Case Study on Weibo," IEEE TRANSACTIONS ON COMPUTATIONAL SOCIAL SYSTEMS, vol. 7, no. 2, pp. 556-562, 2020.

[27] D. Cameron, G. A. Smith, R. Daniulaityte, A. P. Sheth, D. Dave, L. Chen, G. Anand, R. Carlson, K. Z. Watkins and R. Falck, "PREDOSE: A Semantic Web Platform for Drug Abuse Epidemiology using Social Media," J Biomed Inform, vol. 46, no. 6, 2013.
[28] X. Chen, C. Faviez, S. Schuck, A. Lillo-Le-Louët, N. Texier, B. Dahamna, C. Huot, P. Foulquié, S. Pereira, V. Leroux, P. Karapetiantz, A. Guenegou-Arnoux, S. Katsahian, C. Bousquet and A. Burgun, "Mining Patients' Narratives in Social Media for Pharmacovigilance: Adverse Effects and Misuse of Methylphenidate," Frontiers in Pharmacology, vol. 9, no. 541, 2018.

[29] E. C. McNaughton, R. A. Black, M. G. Zulueta, S. H. Budman and S. F. Butler, "Measuring online endorsement of prescription opioids abuse: an integrative methodology," Pharmacoepidemiology and Drug Safety, vol. 21, no. 10, pp. 1081-1092, 2012.

[30] N. AL-TWAIRESH and H. AL-NEGHEIMISH, "Surface and Deep Features Ensemble for Sentiment Analysis of Arabic Tweets," IEEE Access, pp. 84122-84131, 2019.

[31] M. Ebrahimi, A. H. Yazdavar and A. Sheth, "Challenges of Sentiment Analysis for Dynamic Events," IEEE Intelligent Systems, vol. 32, no. 5, 2017.

[32] Microsoft Documentation, "Text Analytics API Documentation," 2021. [Online]. Available: https://docs.microsoft.com/enus/azure/cognitive-services/text-analytics/. [Accessed 3 August 2021].

[33] E. Batbaatar and K. H. Ryu, "Ontology-Based Healthcare Named Entity Recognition from Twitter Messages Using a Recurrent Neural Network Approach," International Journal of Environmental Research and Public Health, vol. 16, no. 3628, 2019.

[34] H. Ren, B. Xu, Y. Wang, C. Yi, C. Huang, X. Kou, T. Xing, M. Yang, J. Tong and Q. Zhang, "Time-Series Anomaly Detection Service at Microsoft," in KDD '19: Proceedings of the 25th ACM SIGKDD International Conference on Knowledge Discovery \& Data Mining, New York, 2019.

[35] R. Zhao, W. Ouyang, H. Li and X. Wang, "Saliency detection by multicontext deep learning," in In Proceedings of the IEEE Conference on Computer Vision and Pattern Recognition, 2015.

[36] J. K. Ord and A. Getis, "Local spatial autocorrelation statistics: distributional issues and an application," Geographical analysis, vol. 27, no. 4, pp. 286-306, 1995.

[37] H.-F. Yu, C.-J. Hsieh, K.-W. Chang and C.-J. Lin, "Large Linear Classification When Data Cannot Fit in Memory," in KDD '10: Proceedings of the 16th ACM SIGKDD international conference on Knowledge discovery and data mining, 2010.

[38] H. Matthies and G. Strang, "The solution of non linear finite element equations," International Journal for Numerical Methods in Engineering, vol. 14, no. 11, p. 1613-1626.

[39] J. Nocedal, "Updating Quasi-Newton Matrices with Limited Storage," Mathematics of Computation, vol. 35, no. 151, p. 773-782, 1980.

[40] NASA Global Landslide Catalog Points (CSV), "The NASA Cooperative Open Online Landslide Repository (COOLR) points, downloadable as a .csv file," 2021. [Online]. Available: https://maps.nccs.nasa.gov/arcgis/home/item.html?id=eec7aee8d2e 040c7b8d3ee5fd0e0d7b9. 
> REPLACE THIS LINE WITH YOUR MANUSCRIPT ID NUMBER (DOUBLE-CLICK HERE TO EDIT) <

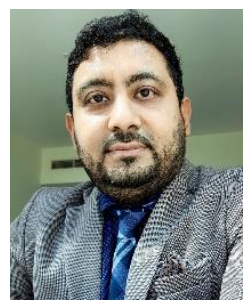

SUFI F. is a Senior AI Solution Architect with Federal Government. He has held Lead Solution Architect roles while working for several federal and state government agencies like Australian Department of Defence, Australian Institute of Family Studies, Victorian Department of Health, Victorian Department of Human Services etc. He has obtained his $\mathrm{PhD}$ in Computer Science \& Information Technology as well as Master of Engineering in Computer Systems from RMIT University, Australia. His research interests include Artificial Intelligence, Machine Learning, Software Development, Big Data Analysis, Cyber and Encryption.

He currently possesses several highly revered industry certifications from Microsoft, The Open Group and others. His current certifications include TOGAF 9 (both Level 1 \& Level 2), ARCHIMATE 3 (both Level $1 \&$ Level 2), Microsoft Certified: Data Analyst Associate, PRINCE 2 (both Foundation \& Practitioner), ITIL V3 and several other Microsoft Certifications in Cloud Technologies. He has published many articles in top ranking Journals like IEEE Journal on Selected Areas in Communication, IEEE Transactions on Information Technology in Biomedicine, IEEE Access and others.

Dr. Sufi's PhD thesis received the best PhD thesis award from the School of Computer Science \& Information Technology, RMIT University in 2011. He has won several awards and scholarships like Australian Post Graduate Award, Victorian Government ICT Post Graduate Scholarship, Commonwealth Government Commercialization Scheme, RMIT SECE School Scholarship, IEEE \& SECE Best Literature Review Award, Victorian Government Certificate of Appreciation etc.

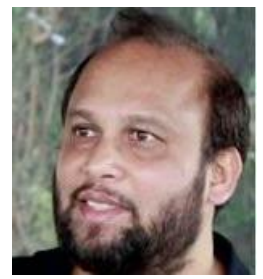

KHALIL I. is a full professor in the School of Science (Computer Science), RMIT University, Melbourne, Australia. Ibrahim obtained his Ph.D. in 2003 from the University of Berne in Switzerland. He has several years of experience in Silicon Valley, California based networking companies as software engineers working on secure network protocols and smart network service provisioning. He worked for EPFL and University of Berne in Switzerland and Osaka University in Japan before joining RMIT University, Australia in 2004.

Ibrahim leads a fairly large research group with approximately $10 \mathrm{PhD}$ students and supervised $21 \mathrm{PhD}$ students towards completions. His research interests are in security and scalable computing in distributed systems such as IoT, Fog, Cloud, Smart Grid, and Remote Health. The specific application areas he is interested in are secure sensor/IoT data analytics, m-health/e- health, wireless body sensor networks, secure IoT applications. Ibrahim and his research team have developed Fully Homomorphic based privacy preserving techniques for Big Data on cloud. This involves data analytics of encrypted data on cloud without any decryption. Since working on encrypted data is extremely time-consuming, Ibrahim's research group have built innovative distributed computing techniques to speed up computations.

Ibrahim received serveral research awards during his career. He is recipient of IEEE LCN 2001 Best Paper award for A Range-Based SLA and Edge Driven Virtual Core Provisioning in DiffServ-VPNs, and Joint author of a Best paper at MobiHealth 2011, Greece. Ibrahim received the prestigious Fritz-Kutter award for the best practice-oriented PhD thesis in Computer Science in whole Switzerland during the academic year 2002/2003. He was interviewed by IEEE Spectrum for the pioneering work on ECG Steganography which was published in IEEE TMBE. (Available online: https://spectrum.ieee.org/techtalk/biomedical/diagnostics/hiding-data-in-a-heartbeat) 